\title{
Load Balancing Algorithm based Adaptive Handovers and Target Cell Load Estimation
}

\author{
Firas Ali Al-Juboori, Ph.D \\ Department of Computer \\ Engineering/University of \\ Baghdad \\ Baghdad, Iraq
}

\author{
Namariq S. AL-Dahwi \\ Department of Computer \\ Engineering/University of \\ Baghdad \\ Baghdad, Iraq
}

\begin{abstract}
The purpose of load balancing in cellular network is to deal with unequal distribution of traffic load over multiple cells. Load balancing is a major issue in LTE (long term evolution) system. A load balancing problem occurs when available wireless resources are not enough to support the requirements of users. Such a problem may lead to blocked or dropped calls and degrade the quality of service. In this paper, we propose a general load -balancing algorithm to help congested cells handle traffic dynamically.
\end{abstract}

\section{Keywords}

LTE system, mobility load balancing, adaptive handover

\section{INTRODUCTION}

3GPP LTE is the next step forward in wireless networks. But it like GSM and WCDMA, still has the problem of load unbalance.[1]

The concept of self-optimizing networks (SON) is introduced In LTE, and the parameter tuning is done automatically

Based on measurements.[2]

The term 'load balancing' can be used to describe any method whereby highly loaded cells share out some of their traffic to less heavily loaded neighbors to make the use of the radio resource more efficient across the whole Network.[3]

Two general methods, channel borrowing and load distributing, have been widely used to solve the load balancing problem. In the first method, overloaded cells try to borrow resources they need from neighboring light loaded cells. But this schemes are not applicable to 3G LTE systems because the channel reuse factor of 3G LTE systems is 1 because of using orthogonal frequency division multiple access (OFDMA) technology. The second algorithms attempt to Distribute the traffic load of the heavy loaded cells by controlling the transmitting power of the base station or

Forcing some mobile stations to handover to neighboring light loaded cells.[4]

There are many ways to re-distribute load among cells. One algorithm is to adjust the cell coverage by modifying the pilot power. A larger pilot power effectively allows more distant mobiles to access the cell, thereby increasing the coverage area. However, automatic adjustment of cell coverage area runs the danger of creating coverage holes. Another way to redistribute the load is to adjust the handover regions between neighboring cells. Such an approach is referred to as

Mobility load balancing (MLB). [3]

a number of algorithms and techniques have been proposed for load balancing in 3GPP LTE. An algorithm to find the suitable handover offset value between the overloaded cell and a possible target cell was proposed. In [5], fuzzy logic controller was envisioned for optimizing handover parameters for adaptive load balancing. Hao Wang et al, proposed a load balancing algorithm that considers heterogeneous services in [6].

In the implementation of the algorithm known as HeaviestFirst Load Balancing Algorithm, each cell receive load status information from its neighbors and compare it with a threshold value. When the load of the eNodeB exceeds a threshold value, the algorithm selects a user that will gain more throughput when transferred to a given target eNodeB. This iterative procedure is repeated until the load of the overloaded cell is reduced to a value equal to or below the threshold value. The algorithm also ensures that the heaviest load is considered first for load balancing. The cell individual power offset (CIPO) parameter, was used for controlling handovers needed to achieve equitable load distribution in [7].

Another algorithm improves the load balancing performance by finding the optimum target cell by using soft computing technique. [8]

In [4] a mobility technique with adaptive handovers based on load balancing was proposed.

In this paper we propose a general load balancing algorithm based on network load status information which is able to automatically indicate optimal adjustments for network parameters.

\section{A network}

The rest of this paper is organized as follows. In section 2 the network model was presented. In Section 3, the problem was formulated. In section 4, the proposed technique was described in detail. In Section 5,the simulation model and results of the proposed technique was presented. Finally the paper is concluded in Section 6.

\section{SYSTEM MODEL}

\subsection{Network Model}

An LTE cellular network is considered as shown in figure 1, there are nine cells numbered with $1, . ., 9$ respectively. each of them is controlled by an eNodeB. Throughout this paper, cell and eNodeB are used interchangeably, and the following assumptions are made:

1) Each user knows instantaneous signal strength from first six tire cells through pilot detection. And all users send them back to their serving eNodeBs periodically.

2) Each eNodeB equally allocates 6 PRB to each user attaching to it.

3) Neighboring eNodeBs exchange their load status information periodically through X2 interface between them. 


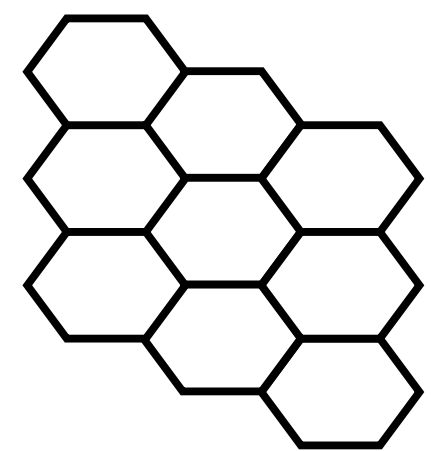

Fig 1: network model.

\subsection{Load Balance Index}

The amount of available resources $v A R(i)$ and the amount of total resources $v T R(i)$ for cell $i(i=0$ means the current serving cell). The ratio of these two values is used for comparison with the predefined thresholds to decide whether the current cell needs load balancing or not.

\section{PROBLEM FORMULATION}

Our object is to make use of enforced handover to balance the load between different cells and keep the network throughput as high as possible at the same time. the problem is that there are cells with heavy load congested cells _ surrounded by cells with light load. So every congested cell will try to shift the overload to its neighboring cells the problem is when congestion occurs in more than one neighboring cell.

\section{PROPOSED TECHNIQUE}

An adaptive handover means that the handover hysteresis thresholds are dynamically updated according to the load conditions of cells. In the conventional handover mechanism, handover decision only depends on degradation of the received signal strength (RSS) of the base station and the hysteresis threshold value is fixed. It does not consider the load status of the current serving cell and the target cell. [4]

The proposed method executes a handover to the best cell for the user with guaranteed high service quality by considering both signal strength and load information. it works as follows:

1. Periodically each cell in the network area exchange load information.

2. According to the current neighbors load information each heavy loaded cell dynamically configures different handover hysteresis thresholds for each neighboring cell. The new handover hysteresis thresholds are calculated by the following Equation:

Newthres (i) $=\alpha \mathrm{i}$ ThHys $(0),(0 \leq \alpha \mathrm{i} \leq 1)$

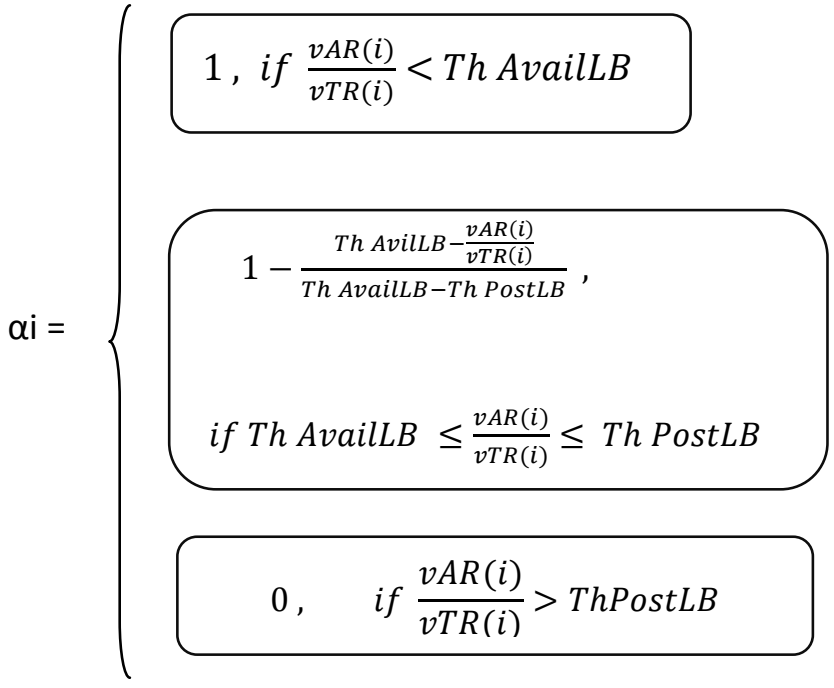

Where the ThHys(0) is the handover hysteresis threshold of the current cell before load balancing. Newthres(i) is

The new handover hysteresis threshold for its neighboring cell

3. Each user sends a measurement report message to his current serving cell periodically. The report message contains the neighbors received radio signal strength.

4. Select the user whose satisfy RSSU1(J)-RSSU1(I)> ThHys $(I, j)$

Where

5. RSSU1(I) :the received signal strength of the serving cell of user $\mathrm{u} 1$.

6. RSSU1(J) :the received signal strength of the neighboring cell of user $\mathrm{u} 1$.

7. To handover from cell I to cell $\mathrm{j}$.

8. Simple load estimation at target cell is made to reduce the number of unnecessary handover. this is done by using network manager who add the hand over calls to the load of the target cell, if the target cell falls in the overloaded status the this hand over calls blocks and search for new target cell from the candidate target cells pool, else this operation complete.

9. The overloaded cell executes the load balance algorithm until its load status return to the light load condition.

\section{SIMULATION MODELS AND RESULTS}

The proposed mobility management system Was designed and implemented in the matlab, in order to show the improvement of the proposed load balancing algorithm , three simulation systems was designed:

1. A system without load balancing.

2. A system only with load balancing.

3. A system with load balancing using target cell load estimation. 
* New call blocking rate: Number of blocked calls/total number of new calls.

* Handover number: The number of handover calls

* Handover call blocking rate: Number of blocked handover calls / the total number of handover calls.

The simulation model of the cellular network area consists of 9 macro cells with the same radius of $1 \mathrm{~km}$ as shown in figure 1. The users distributed randomly in the cells. Various parameters for the simulation are Described in table 1:

Table 1. simulation parameters

\begin{tabular}{|c|c|}
\hline parameters & values \\
\hline Cell Number & 21 \\
\hline Cell Diameter & $1000 \mathrm{~m}$ \\
\hline Cell Capacity & $0 \sim 130$ PRBs \\
\hline UE Number & Random waypoint \\
\hline UE Mobility & $0 \sim 100 \mathrm{~km} / \mathrm{h}$ \\
\hline UE Speed & Mean: $3 \sim 8$ PRBs \\
\hline Traffic Model & $5 \mathrm{~dB}$ \\
\hline Normal Handover Threshold & $0 \sim 5 \mathrm{~dB}$ \\
\hline Dynamic Handover & 0.2 \\
\hline Threshold & 0.3 \\
\hline Th $_{\text {pre-LB }}$ & 0.4 \\
\hline Th $_{\text {Avail-LB }}$ & $T_{\text {post-LB }}$ \\
\hline
\end{tabular}

Using matlab to simulate the mobile network, the users randomly distributed on the nine cells, then periodically calls the load balancing algorithm for over loaded cells. the hand over calls blocking rate reduce using load balancing algorithm ,but using estimation the hand over calls blocking rate increased because the many of target cells prevent the users from entering them coverage area. The figure below show the relation between handover blocking rate and user number.

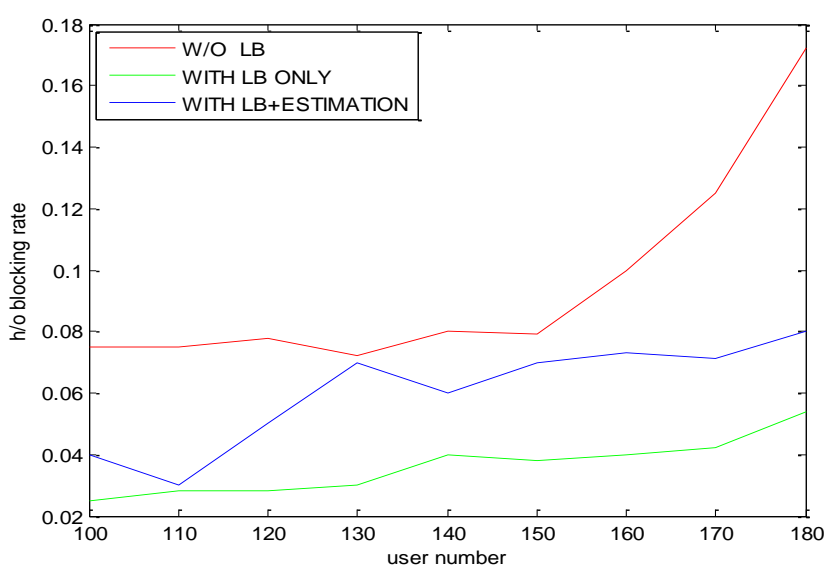

Fig 2: user No. with $\mathbf{h} / \mathrm{o}$ blocking rate
The number of new calls blocking rate for proposed algorithm is less than the conventional one because the load is distributed from over loaded cell to them neighbors light loaded cells so many of physical resource block be free and be able to accommodate new calls

But using estimation the new calls block slightly be more than without estimation because many of new calls not accepted from cells which estimated to be over loaded cells if they accept these new calls.

Figure below shows the relation between the new calls blocking rate and the user number.

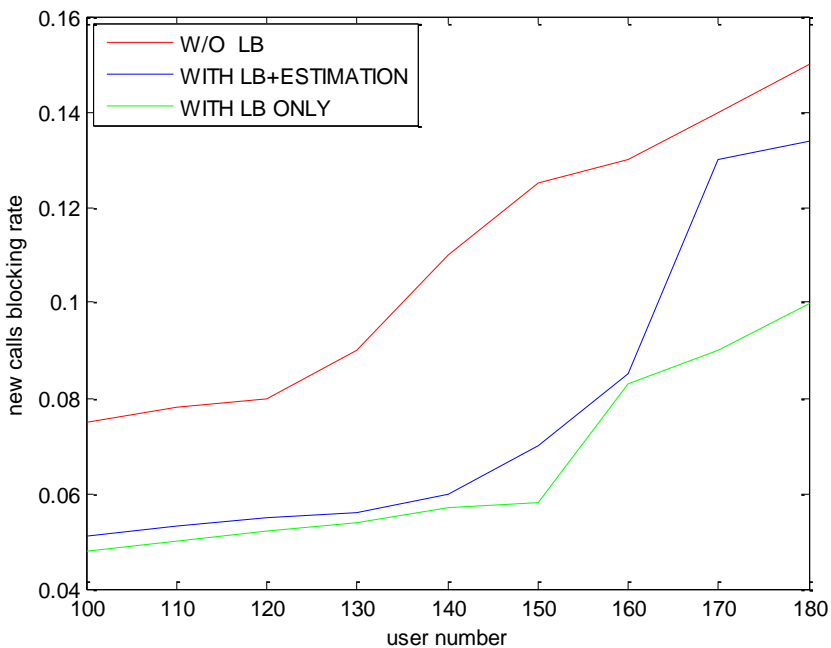

Fig 3: user No. with new call blocking rate

The hand over number is increases as the number of user terminals is increased as shown in the figure below:

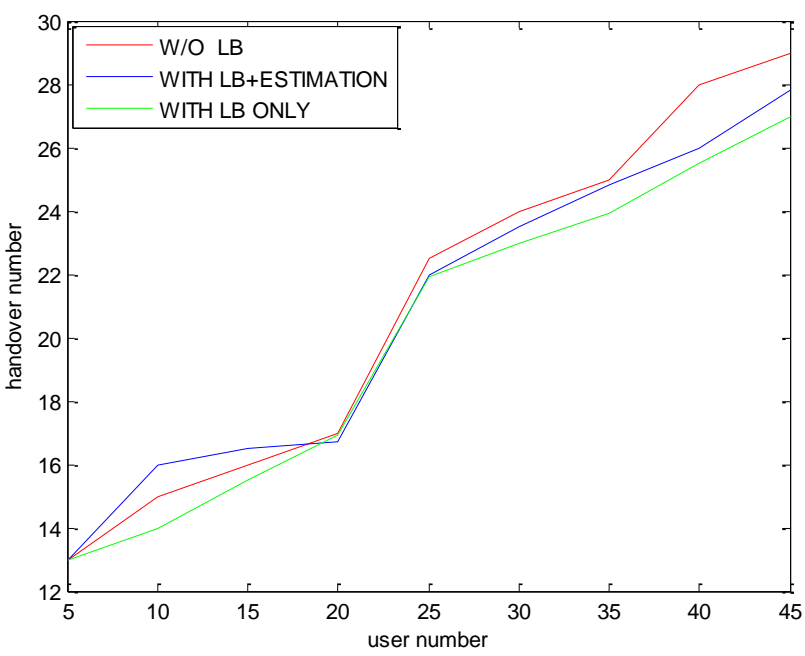

Fig 3: user No. with handover number

\section{CONCLUSION}

Our In this paper a mobility management technique with adaptive handovers based on load balancing for LTE cellular system was proposed, load estimation at the target cell was used to reduce the number of unnecessary hand over. 
For future work develop better estimation techniques in order to select better target cell for adaptive handovers.

\section{REFERENCES}

[1] Hao Wang, Lianghui Ding, Ping Wu, Zhiwen Pan, Nan Liu,Xiaohu You, 2010, "Dynamic Load Balancing in 3GPPLTE Multi-Cell Networks with Heterogenous Services," IEEE.

[2] Andreas Lobinger, Szymon Stefanski, Thomas Jansen, Irina Balan,2011," Coordinating Handover Parameter Optimization and Load Balancing in LTE SelfOptimizing Networks," IEEE.

[3] Raymond Kwan, Rob Arnott, Robert Paterson, Riccardo Trivisonno, Mitsuhiro Kubota,2010. " On Mobility Load Balancing for LTE Systems," IEEE.

[4] Qi-Ping Yang, Jae-Woo Kim, Tae-Hyong Kim, 2012." Mobility Prediction and Load Balancing Based Adaptive Handovers for LTE Systems," IJCSE Vol. 4, No. 04
[5] Rodriguez J., de la Bandera I., Munoz P., and Barco R., 2011. "Load Balancing in a Realistic Urban Scenario for LTE Networks," IEEE 73rd Vehicular Technology Conference (VTC Spring), pp. 1-5

[6] Hao Wang et al. 2010."Dynamic Load Balancing in 3GPP LTE Multi-Cell Networks with Heterogenous services", ICST Conference, Beijing.

[7] Manfred R., Jakob B., Paul A., and Wilfried W., 2009. "Ruled-based Algorithms for Self-x Functionalities in Radio Access Networks," Conference Proceedings of ICT-Mobile Summit.

[8] Aderemi A. and Matthew K. 2012. "Adaptive NeuroFuzzy Inference System for Dynamic Load Balancing in 3GPP LTE", International Journal of Advanced Research in Artificial Intelligence, Vol. 1, No. 1 